\title{
Hepatocellular Carcinoma Recurrence with Metachronous Peritoneal Metastasis - A Controversial
} Surgical Indication

\author{
João Simões ${ }^{1,2 *}$, Maria João Amaral ${ }^{1,2}$, Marco Serôdio ${ }^{1,2}$ and José Guilherme Tralhão $o^{1,2,3}$ \\ ${ }^{1}$ General Surgery Department, Centro Hospitalar e Universitário de Coimbra, Coimbra, Portugal \\ ${ }^{2}$ Faculty of Medicine, University of Coimbra, Coimbra, Portugal \\ ${ }^{3}$ Faculty of Medicine, Coimbra Institute for Clinical and Biomedical Research (iCBR) area of Environment Genetics and \\ Oncobiology (CIMAGO), University of Coimbra, Coimbra, Portugal
}

\begin{abstract}
Intraperitoneal metastases of a non-ruptured HCC are rare. Metastatic disease is an expression of an advanced disease, is associated with poor prognosis and can occur at the time of hepatic recurrence.

We present the case of a female patient who underwent surgery for a big hepatic adenoma that suffered malignant transformation. Fifteen months later, she had HCC liver recurrence with peritoneal metastases, being submitted to a new surgery with complete resection of the hepatic mass and peritoneal implants.

In the absence of HCC rupture, peritoneal dissemination is not common. Aggressive surgical treatment and adjuvant treatment with sorafenib could prolong patients' overall survival but there is still no standard management for these patients.
\end{abstract}

Keywords

Hepatocellular carcinoma, Hepatic adenoma, Recurrence, Peritoneal metastases

\section{Introduction}

Hepatocellular carcinoma (HCC) is the fifth most common solid tumor worldwide and the most common primary liver cancer, with incidence increase in developed countries $[1,2]$. There are many documented risk factors for HCC: Viral hepatitis, cirrhosis due alcoholic and nonalcoholic disease, toxins, drugs and metabolic diseases like hereditary hemochromatosis, among others [3].

HCC can also arise from hepatic adenomas. Hepatic adenoma is a benign tumor of the liver that results from monoclonal proliferation of hepatocytes. The use of estrogencontaining oral contraceptives is a well-known risk factor for hepatic adenoma development. The risk of malignant transformation from hepatic adenoma to HCC is very low (4$5 \%)$ and is related to adenoma size [4].

Intraperitoneal metastases of a non-ruptured HCC are rare, with an incidence of $2-16 \%[1,3,5]$. Metastatic disease is an expression of an advanced disease, is associated with poor prognosis and can occur at the time of hepatic recurrence $[2,6,7]$. There is no recognized standard treatment for this condition, although there are some described cases of patients treated with chemotherapy (sorafenib) or surgery (resection, cytoreductive surgery, hyperthermic intraperitoneal chemotherapy), which could contribute to prolong survival $[2,6]$. On the other hand, palliative treatment only reaches an overall survival (OS) of 6-14 months [8,9].

We present a case report of a female patient that underwent surgery for a growing hepatic adenoma. Pathological analysis showed a hepatocellular carcinoma and fifteen months later she presented with $\mathrm{HCC}$ recurrence with peritoneal metastases.

*Corresponding author: João Simões, General Surgery Department, Centro Hospitalar e Universitário de Coimbra, Coimbra, Portugal; Faculty of Medicine, University of Coimbra, Coimbra, Portugal

Accepted: December 24, 2021

Published online: December 26, 2021

Citation: Simões J, Amaral MJ, Serôdio M, et al. (2021) Hepatocellular Carcinoma Recurrence with Metachronous Peritoneal Metastasis - A Controversial Surgical Indication. J Gastrointest Hepat Surg 4(1):46-50 
Citation: Simões J, Amaral MJ, Serôdio M, et al. (2021) Hepatocellular Carcinoma Recurrence with Metachronous Peritoneal Metastasis A Controversial Surgical Indication. J Gastrointest Hepat Surg 4(1):46-50

\section{Case Report}

A 41-year-old caucasian female patient was diagnosed with a $4 \mathrm{~cm}$ hepatic solid mass on her left liver, consistent with an adenoma, in a routine abdominal ultrasound. She had a past medical history of irritable bowel syndrome and asthma. She was asymptomatic. There was no recent history of loss of appetite, weight loss, abdominal pain or altered bowel habits. She had a family history of gastric cancer (father), primary biliary cirrhosis (mother) and hepatic, gastric and colorectal cancer from her mother's side of the family.

After the diagnosis, the patient was advised to stop taking the oral contraceptive pill (cyproterone with ethinylestradiol) that she had been taking since she was 31 years old, and to repeat abdominal ultrasound periodically for control. On a routine ultrasound, 12 months later, the adenoma had grown up to $11 \mathrm{~cm}$.

There were no radiologic signs of cirrhosis and blood tests and tumor markers were always within normal values. Hepatitis B and C infections were excluded. There was no diabetes mellitus, hypertension or dyslipidemia suggesting metabolic syndrome with is associated with nonalcoholic steatohepatitis. She also denied alcohol consumption.

The patient was evaluated in a General Surgery consultation and the case presented in a Multidisciplinary Hepatobiliopancreatic Meeting (MHBPM). The patient was submitted to a laparoscopic hepatic subsegmentectomy (S2 and S3), without rupture of the mass or postoperative complications. The pathology report showed a moderately differentiated hepatocellular carcinoma (G2) with $11 \times 6.5 \times$ $4.5 \mathrm{~cm}$, with a resection margin of $0.3 \mathrm{~cm}$, without signs of rupture or chronic hepatic disease (Figure 1). Neoplastic cells were negative to CK19. Lymphovascular invasion was present and it was classified as a pTNM T1bNOMO (according to the $8^{\text {th }}$ edition of the American Joint Committee on Cancer). Alpha-fetoprotein (AFP), carcinoembryonic antigen (CEA) and carbohydrate antigen 19.9 (CA19.9) were within normal values. Once again, the case was presented at a MHBPM and the decision was continued surveillance.

The first hepatic magnetic resonance imaging (HMRI) after surgery was performed 6 months later, without any signs of recurrence or metastasis. Fifteen months after surgery, a new HMRI showed a $17 \mathrm{~mm}$ mass in the left liver, near the hepatic resection parenchyma, with T2 hypersignal (Figure 1), without evidence of enhancement after contrast and without restriction diffusion-weighted images (Figure 2). Also, it showed a nodular formation on Morison's space and other one near the left hepatic lobe, with $\mathrm{T} 2$ hypersignal, suggesting peritoneal metastasis. There were no other changes in the liver parenchyma, biliary tree or peritoneal fluid. Tumor markers remained within normal values (AFP $1.9 \mathrm{ng} / \mathrm{mL}$, CEA $<0.5 \mathrm{ng} / \mathrm{mL}$ and CA $19.9<2.0 \mathrm{U} / \mathrm{mL}$ ).

After a new case discussion at a MHBPM, with the presumptive diagnosis of peritoneal relapse and a liver nodule of unknown etiology, the patient underwent an exploratory laparoscopy that revealed the already known solid nodule on Morison's space and on the great omentum, and a solid mass on the left hepatic lobe, near the previously resected area. Intraoperative pathology results confirmed peritoneal metastasis of HCC (Figure 3 and Figure 4). There was no peritoneal effusion and we proceeded to a laparoscopic omentectomy and a new hepatic subsegmentectomy of the

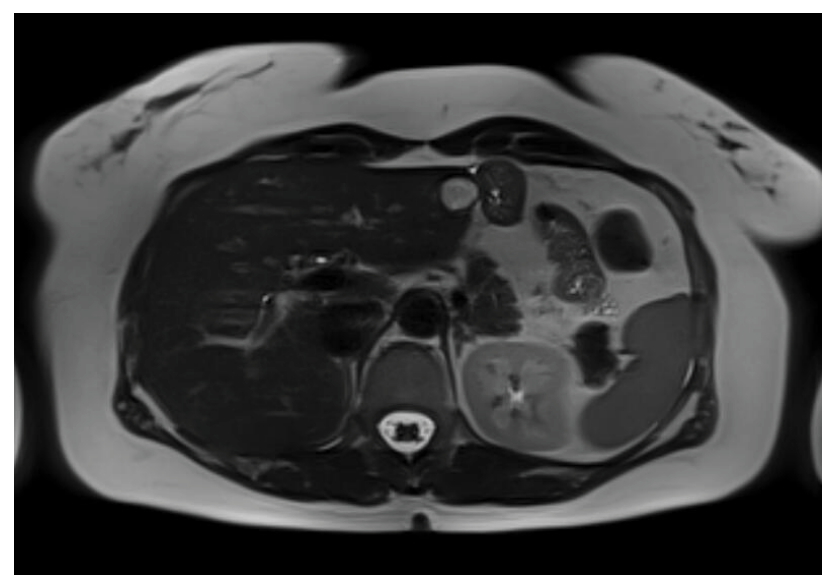

Figure 1: MRI (axial view), T2, revealing hyperintense $17 \mathrm{~mm}$ mass in the left liver, near the hepatic resection parenchyma.

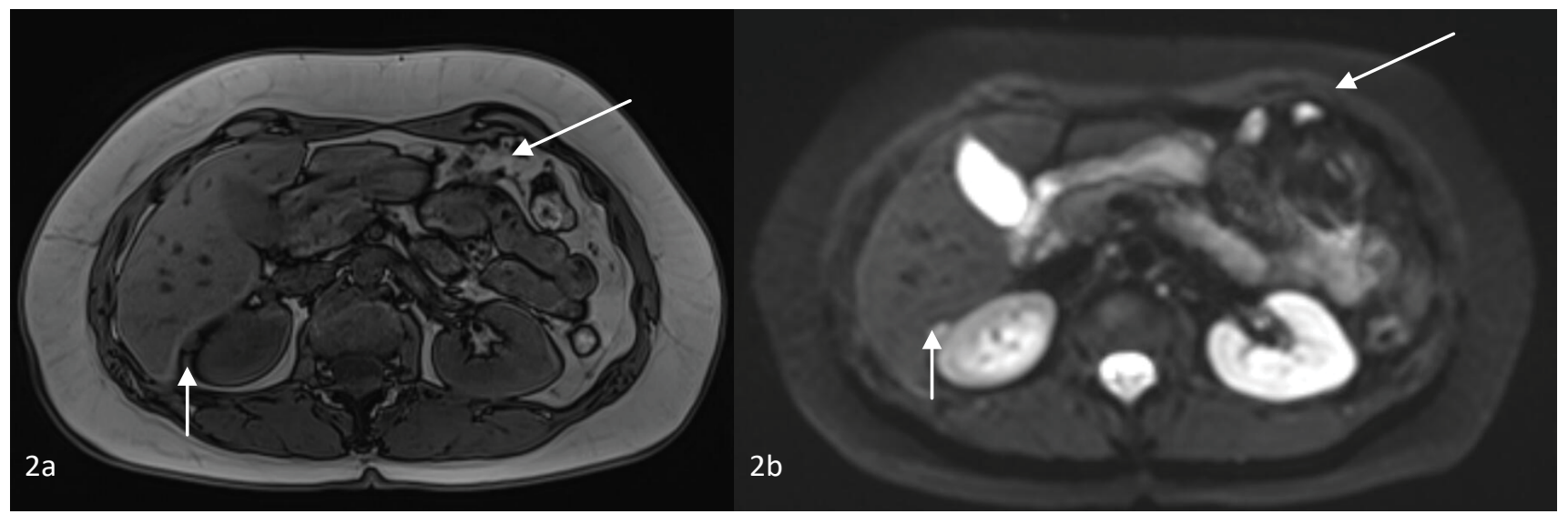

Figure 2: MRI (axial view), opposed phase (2a) and restricted diffusion phase (2b), revealing peritoneal carcinomatosis on Morrison space and great omentum (arrows). 
Citation: Simões J, Amaral MJ, Serôdio M, et al. (2021) Hepatocellular Carcinoma Recurrence with Metachronous Peritoneal Metastasis A Controversial Surgical Indication. J Gastrointest Hepat Surg 4(1):46-50

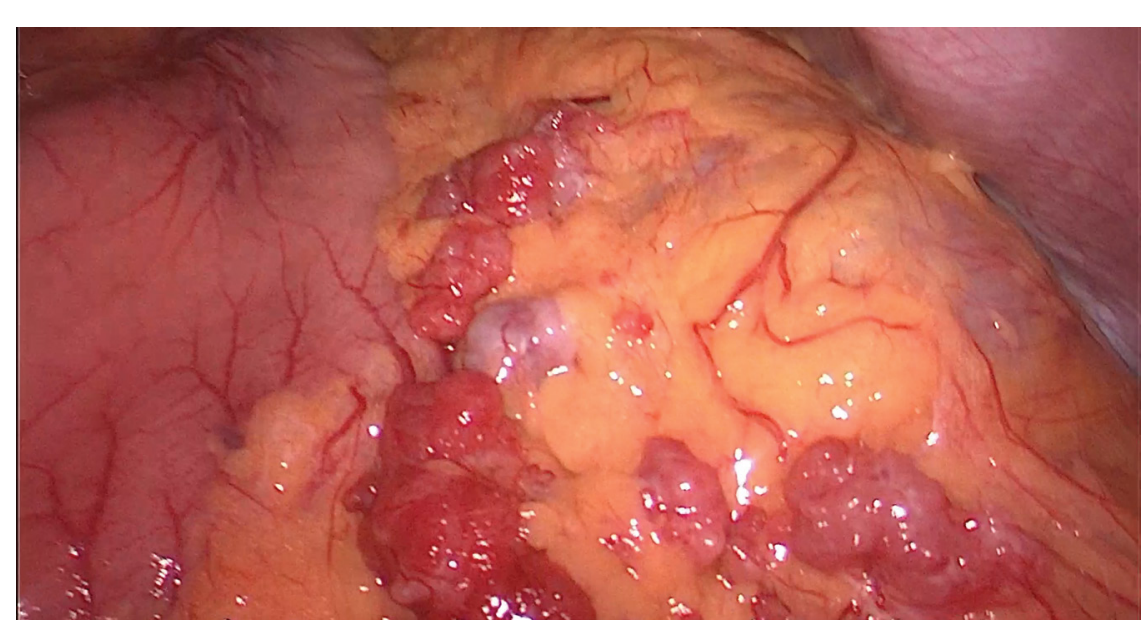

Figure 3: Peritoneal carcinomatosis in great omentum.

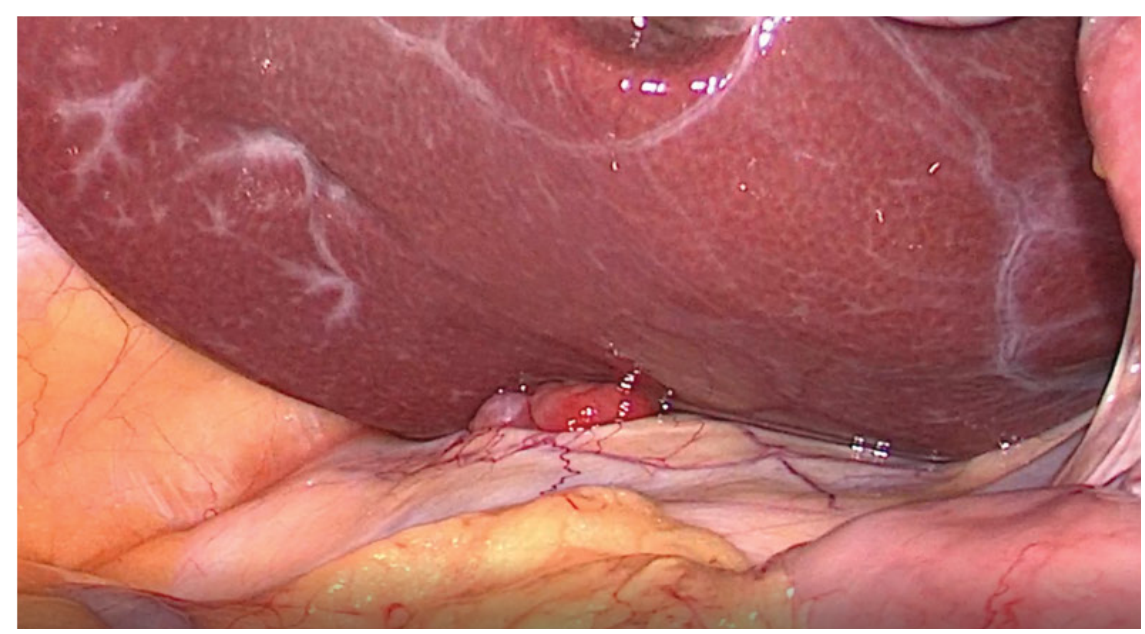

Figure 4: Peritoneal carcinomatosis on Morison's space.

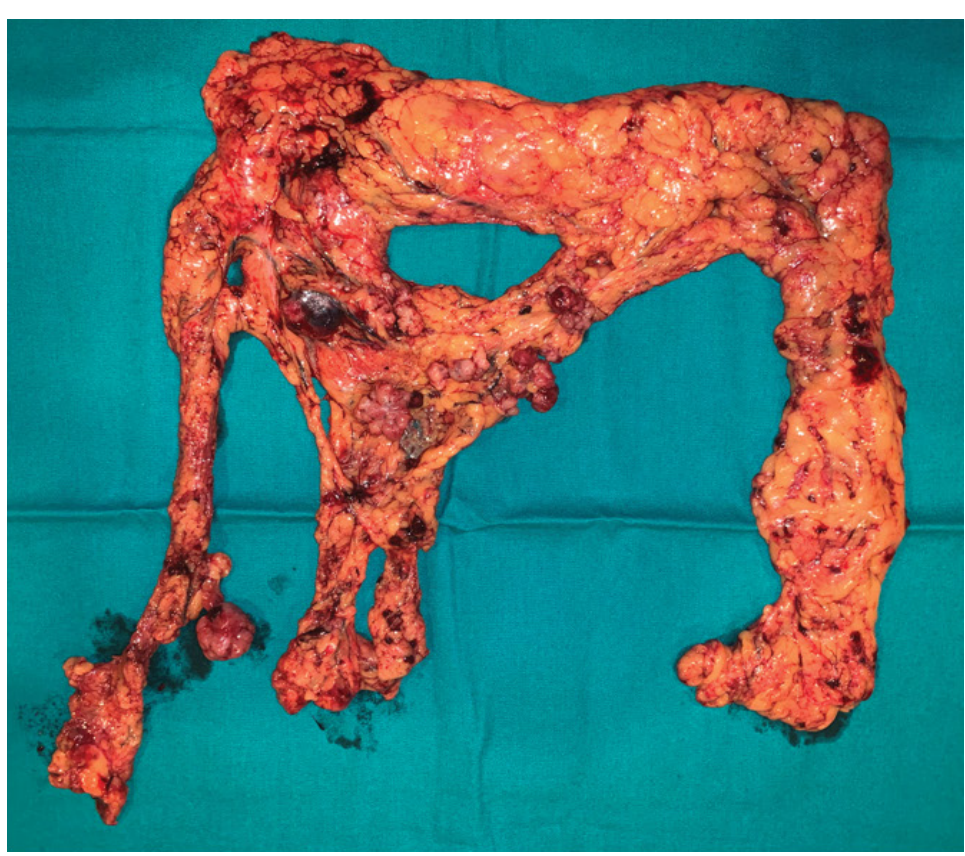

Figure 5: Greater omentum with peritoneal carcinomatosis. 


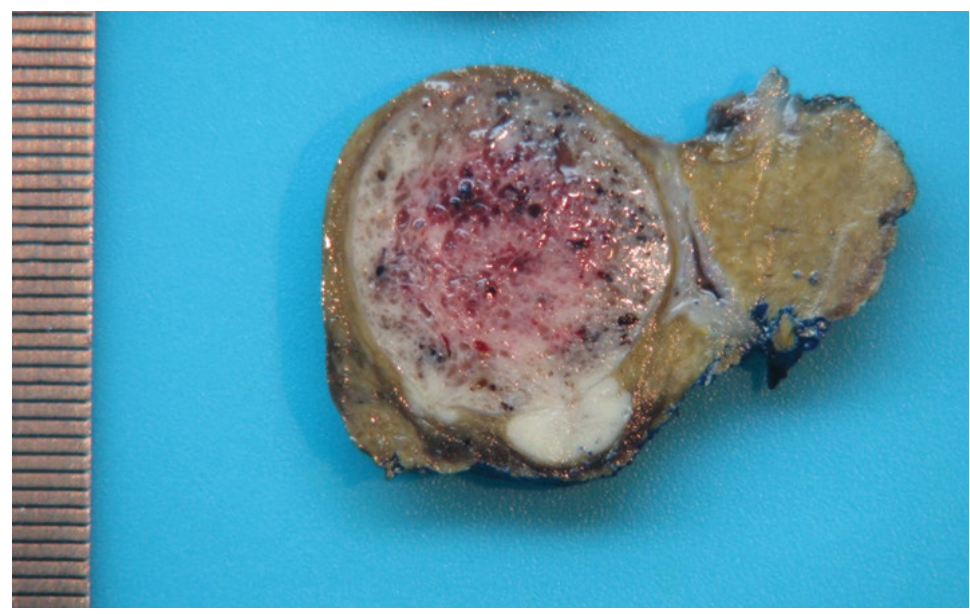

Figure 6: Liver specimen after hepatic subsegmentectomy showing soft white HCC with haemorrahge.

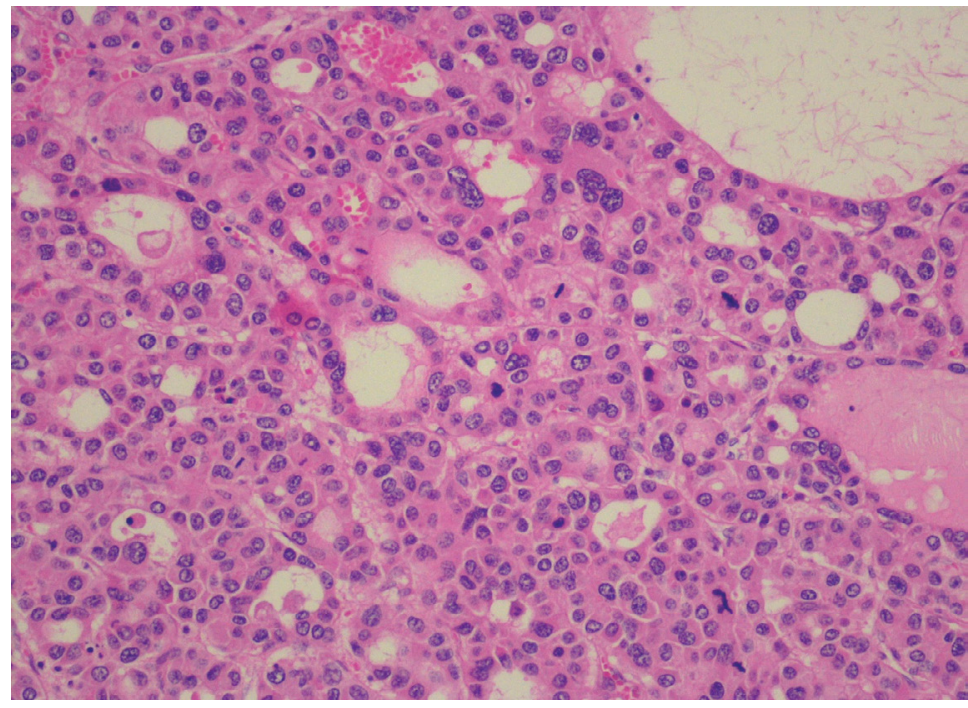

Figure 7: Poorly differentiated carcinoma with trabecular and acinar patterns showing tumor cells arranged in pseudoglandular formations with lumina containing eosinophilic material $(\times 200)$. Neoplastic cells shows eosinophilic cytoplasm and pleomorphic nuclei, with several mitotic figures.

segments 2 and 3 (Figure 5 and Figure 6). The postoperative period was uneventful and the patient was discharged on postoperative day 2 .

The anatomopathological results revealed a HCC relapse, poorly differentiated (G3), without lymphovascular invasion (Figure 7), and peritoneal metastases from HCC localized on the great omentum. It was classified as PT1bNOM1 (according to $8^{\text {th }}$ edition of the American Joint Committee on Cancer). On the MHBPM, adjuvant treatment with sorafenib was proposed, $400 \mathrm{mg}$ bid, which the patient accepted.

\section{Discussion}

HCC is a well-known disease in the cirrhotic liver. Data in non-cirrhotic liver is limited and these cases represent less than $20 \%$ of all HCC [3]. Hepatic adenoma is a benign condition that develops mostly in normal livers of young women taking oral contraceptive pills containing estrogen. In near $5 \%$ of cases, hepatic adenomas can transform into HCC $[4,10]$.
Extrahepatic disease is more common in patients with noncirrhotic liver. HCC metastases are more common in the liver, lungs, portal vein, bones and regional lymph nodes, followed by the gastrointestinal tract, adrenal glands, peritoneal surface, skin, brain and muscle. Risk factors for metastasis are large tumor size, multifocality, vascular invasion and poor histologic differentiation $[3,11]$.

There are several reports of peritoneal metastasis after HCC rupture into the peritoneal cavity, which seeds metastatic deposits. Also, peritoneal metastasis can occur after percutaneous procedures in 0.9 to $12.5 \%$ of cases and be due to locoregional lymph node metastasis $[2,7,12]$. On the other hand, in the absence of HCC rupture, metastatic dissemination has an incidence of $2-16 \%$ after hepatic resection. As mentioned before, there is no standard treatment to this condition and it this is considered an advanced stage of the disease $[5,9]$.

Peritoneal metastasis from HCC can be synchronous or 
Citation: Simões J, Amaral MJ, Serôdio M, et al. (2021) Hepatocellular Carcinoma Recurrence with Metachronous Peritoneal Metastasis A Controversial Surgical Indication. J Gastrointest Hepat Surg 4(1):46-50

metachronous and are usually a unique implant $[1,3,8,13]$. Omental seeding is a less common form of presentation. Patients with peritoneal metastasis of HCC can reach a mortality rate as high as $50 \%$ and metachronous presentation has better prognosis than synchronous presentation $[2,9]$.

On the first surgery there was no rupture of the specimen inside the abdomen, minimal resection margin was $0.3 \mathrm{~cm}$ and the histopathology revealed a G2 HCC. Peritoneal metastases are more commonly seen in $\mathrm{G} 2$ and $\mathrm{G} 3 \mathrm{HCC}$, which could mean that peritoneal disease is associated with biological features of the primary HCC [6]. Microvascular invasion is commonly seen in moderately and poor-differentiated HCC and is associated with aggressive tumor biology, metastatic spread and hepatic recurrence [11].

Recurrent liver disease is associated with poor prognosis, but successful treatment of intrahepatic HCC provides a survival benefit. Also, resection of peritoneal metastases may improve survival rate, especially if they are localized [12]. In the case of our patient, we reached a complete resection of macroscopic disease, both the liver recurrence and peritoneal metastases, by performing total omentectomy.

Surgery could be a good approach to localized peritoneal metastasis of HCC in patients with a controlled primary disease and may improve survival in selected patients, in the absence of other extrahepatic metastases and who are suitable for radical treatment. Recurrence is not rare in visceral peritoneal disease but decreases after en bloc resection of parietal peritoneal disease $[6,12]$.

Lin, et al. showed that patients submitted to surgical resection of HCC metastases have better median survival time than those in whom resection was not made $[1,3]$. Mehta, et al. describes a median overall survival (OS) of 46.7 months with a 5 -year survival rate of $49.4 \%$ after aggressive surgical treatment in patients with $\mathrm{HCC}$ and peritoneal metastasis [8]. On the other hand, incomplete resection has a shorter OS time [9].

Some studies report the role in cytoreductive surgery (CRS) and hyperthermic intraperitoneal chemotherapy (HIPEC). CRS + HIPEC are indicated for colorectal peritoneal metastases and for some primary peritoneal malignancies [8]. CRS has the aim to remove all macroscopic metastatic peritoneal disease and HIPEC can have a cytotoxic effect on residual microscopic disease. Zhong-He, et al. presented a case of a patient with synchronous peritoneal metastasis of HCC, treated with CRS + HIPEC and 6 cycles of adjuvant normothermic intraperitoneal and intravenous chemotherapy (cisplatin and docetaxel), because he was not responding to sorafenib; after this treatment, he was disease-free for over 22 months [9]. Other study showed that, in selected cases of HCC peritoneal metastases, CRS + HIPEC has better median OS than CRS alone, but without statistical significance [6].

Treatment with sorafenib could be an option as a postoperative adjuvant in selected patients with extrahepatic metastases, although it is still not considered standard therapeutic on every case, because it is not an innocuous drug [2].

Most studies that showed some benefit in the treatment of patients with peritoneal disease from HCC, with systemic chemotherapy or radical surgery, are retrospective studies, thus it is essential to draw prospective randomized studies in order to clarify the adequate management for these patients.

\section{Conclusion}

Hepatic adenoma transformation to HCC is uncommon. Also, peritoneal metastases from a non-ruptured HCC are rare and have a poor prognosis. Their development can be associated with biological features of the primary HCC. Excision, cytoreductive surgery, HIPEC or systemic chemotherapy with sorafenib could prolong patients' overall survival but there is still no standard treatment. For this reason, there is an urgent need for investigating in this field and innovate treatment of $\mathrm{HCC}$ with peritoneal metastases.

\section{References}

1. Lin CC, Liang HP, Lee HS, et al. (2009) Clinical manifestations and survival of hepatocellular carcinoma patients with peritoneal metastasis. J Gastroenterol Hepatol 24: 815-820.

2. Chua TC, Morris DL (2011) Exploring the role of resection of extrahepatic metastases from hepatocellular carcinoma. Surg Oncol 21: 95-101.

3. Herath $\mathrm{H}$, Kulatunga $A$ (2017) Large hepatocellular carcinoma in a non-cirrhotic liver with peritoneal and omental metastasis in a healthy man: A case report. J Med Case Rep 11: 34.

4. Mathew R, Manolea F, Girgis S, et al. (2019) Malignant transformation of hepatic adenoma complicated by rupture and hemorrhage: An extremely rare clinical entity. Intractable Rare Dis Res 8: 266-270.

5. Ding J, Chua TC, Al-Mohaimeed K, et al. (2010) Hepatocellular carcinoma peritoneal metastases: Report of three cases and collective review of the literature. Annals Academy of Medicine 39: 734-737.

6. Berger $Y$, Spivak JH, Heskel M, et al. (2016) Extrahepatic metastasectomy for hepatocellular carcinoma: Predictors of long-term survival. J Surg Oncol 114: 469-474.

7. Portolani N, Baiocchi GL, Gheza F, et al. (2014) Parietal and peritoneal localizations of hepatocellular carcinoma: is there a place for curative surgery? World J Surg Oncol 12: 298.

8. Mehta S, Schwarz L, Spiliotis J, et al. (2018) Is there an oncological interest in the combination of CRS/HIPEC for peritoneal carcinomatosis of HCC? Eur J Surg Oncol 44: 1786-1792.

9. Ji Z, An SJ, Li XB, et al. (2019) Long-term progression-free survival of hepatocellular carcinoma with synchronous diffuse peritoneal metastasis treated by CRS+HIPEC: A case report and literature review. Medicine 98: e14628.

10. Rebouissou S, Nault JC (2020) Advances in molecular classification and precision oncology in hepatocellular carcinoma. J Hepatol 72: 215-229.

11. Zimmermann A (2017) Invasion patterns and metastatic patterns of hepatocellular carcinoma. In: Tumors and tumor-like lesions of the hepatobiliary tract. Springer International Publishing, Switzerland.

12. Takemura N, Hasegawa K, Aoki T, et al. (2014) Surgical resection of peritoneal or thoracoabdominal wall implants from hepatocellular carcinoma. Br J Surg 101: 1017-1022.

13. Mesa A, Prieto A, Fuentes-Martínez N, et al. (2014) Peritoneal carcinomatosis secondary to spread of hepatocellular carcinoma originated upon a healthy liver. Rev EspEnferm Dig 106: 53-54. 